\title{
Neue Materialien zur Beseitigung von Ölverschmutzungen
}

\author{
Michael Herzog, Sanchi Nenkova, Radka Garvanska, Valeria Gancheva
}

\section{Zusammenfassung}

Der Beitrag berichtet von der Entwicklung eines als Absorptionsmittel geeigneten Kompositmaterials auf der Basis von verschiedene Zellulosestrukturen aufweisenden Ausgangsstoffen und einem Bindemittel. Solche Absorptionsmittel sind einsetzbar für die Aufnahme von Mineralölprodukten aus wässrigen Phasen, beispielsweise aus belasteten Oberflächengewässern. Hierzu wurden geeignete biogene Ausgangsstoffe identifiziert sowie ein Verfahren zur Herstellung von Sorptionsmitteln entwickelt und dessen prinzipielle Eignung demonstriert.

\section{Abstract}

In this paper we report on the development of composite materials applicable as absoption agent based on cellulose of special structures and a binder.

Such agents may used for the take-up of mineral oils from aqueous phases like contaminated suface water. To this end, suitable raw materials and processing parameters were established to demonstrate the properties and performance of the products.

\section{Einleitung}

Ölverschmutzungen von Gewässern greifen schon bei geringen Kontaminationen störend in die vielfältigen Stoffwechselprozesse in natürlichen und anthropogenen Lebensräumen ein und stellen noch immer offensichtliche und als besonders besorgniserregend wahrgenommene Umweltbelastungen dar.

Durch Tankschiffe verursachte Ölkatastrophen haben sich im Verlauf der letzten 30 Jahren signifikant reduziert [Huijer 2005]. Wie Abbildung 1 zeigt, betrug die durchschnittliche Anzahl noch in den 70er Jahren ein Vielfaches im Vergleich zur gegenwärtigen Dekade. Dieser Trend ist dem zumindest seit 1985 stetig ansteigenden Seehandel mit Erdöl bzw. Mineralölprodukten entgegengesetzt und spiegelt den Erfolg vielfältiger internationaler Bemühungen zur Vermeidung des Eintrags von wassergefährdenden Stoffen wider.

Seit dem Unglück der »Prestige« vor der spanischen Küste im November 2002, bei dem von den $70.000 \mathrm{t}$ Schweröl an Bord ein großer Teil in den Atlantik austrat und hunderte Kilometer Küste verschmutzte, sind weltweit keine Störfälle vergleichbarer Größenordnung zu verzeichnen gewesen.

Allerdings verursachen Unfälle auch heute noch großflächige Schädigungen von aquatischen Biotopen und haben mit den erforderlichen Reinigungsmaßnah- men in Küstenregionen und der Beeinträchtigung von Fischerei und industrieller Wassernutzung drastische Auswirkungen und verursachen erhebliche volkswirtschaftliche Schäden.

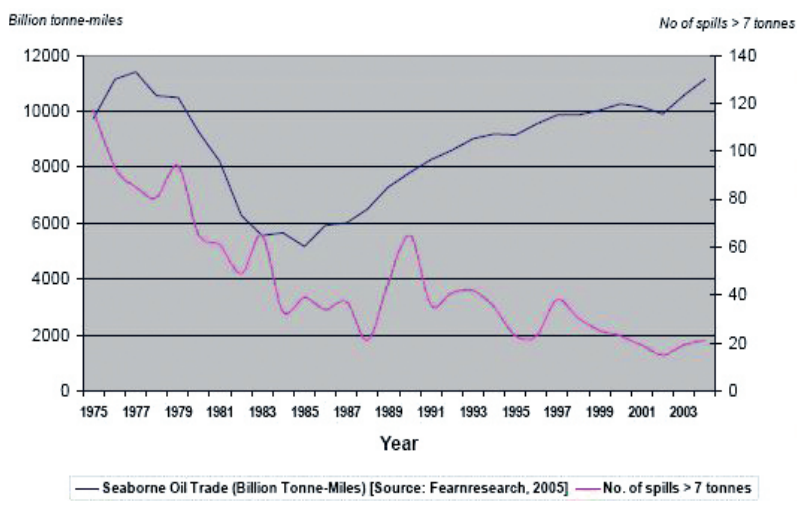

Abb. 1: Vergleich von Transportleistung und Anzahl größerer Tankerunfälle [Huijer 2005]

Während die Belastung der Weltmeere durch spektakuläre Tankerunfälle seit den 70er Jahren deutlich zurückgegangen ist, stammt der überwiegende Teil der in Gewässer gelangten Mineralöle heute aus relativ kleinteiligen Quellen im Bereich der küstennahen wirtschaftlichen Aktivitäten mit im Einzelfall nur geringem Eintrag von Schadstoffen in aquatische Systeme [UN 1993].

Übliche, oft chronische Ölverschmutzungen wie sie überall in den Küstengewässern in der Nähe von 
Industriegebieten oder Schifffahrtswegen auftreten, beschränken sich im Allgemeinen auf relativ dünne Ölfilme, die auf der Wasseroberfläche schwimmen und nicht unmittelbar mit der Vegetation in Tiefen unter etwa einem halben Meter in Berührung kommen [Schramm 1972].

Für länger zurückliegende Katastrophen wie den Unfall der »Exxon Valdez« im Prince William Sound im März 1989 sind das Ausmaß der Belastungen sehr detailliert dokumentiert und auch die Langzeitwirkungen erfasst worden. Hierbei hat sich gezeigt, dass eine weitgehende Erholung der natürlichen Systeme bereits in relativ kurzen Zeiträumen erfolgt [Exxon 2008]. Dem tieferen Verständnis der Ausbreitungsmechanismen und der Konzeption von Schutz- und Vorsorgemaßnahmen dienten Arbeiten zur Modellierung von Ölfreisetzungen [Li 2007]. Maßnahmen wie starre und flexible Barrieren sind für verschiedene Bedingungen hinsichtlich Strömung und Wellengang mit dem Ziel modelliert worden [Amini et al. 2008], eine möglichst vollständige Entfernung von aufschwimmenden Leichtölen zu erreichen.

Dies betrifft vor allem sensible bzw. schützenswerte Naturräume; insbesondere stellen die Auswirkungen auf industrielle Anlagen wie Meereswasserentsalzungsanlagen für die Trinkwasserversorgung sowie landwirtschaftliche und industrielle Verbraucher eine unmittelbare Gefährdung für urbane Siedlungsräume dar [Elshorbagy/Elhakeem 2008].

Ferner wurden einige gesetzliche Maßnahmen wie der Oil Pollution Act erlassen, die mit Doppelhüllentankern und Zuverlässigkeitsprüfungen wesentliche Elemente zu einer maßgeblichen Minimierung des Gefahrenpotenzials in den 90er Jahren beigetragen haben [Homan/Steiner 2008]. Solche Maßnahmen sind auch in ihrer wirtschaftlichen Effektivität bewertet worden [Vanem et al. 2008]. Für eine umfassende Darstellung sei auf die Arbeit von Ornitz und Champ [2002] verwiesen.

Mit der Entschließung der Europäischen Gemeinschaft zur Erstellung eines Aktionsprogramms auf dem Gebiet der Überwachung und Verringerung der Ölverschmutzung des Meeres wurde transnational gravierenden Störfällen begegnet [EU 1978].

Dies beinhaltet die Reduzierung der Gefährdung und Minimierung der Auswirkungen durch verbindliche technische Anforderungen und eine klare Regelung der Verantwortlichkeiten für die Vorsorge und Gefahrenabwehr sowie klare Szenarien für Aktionen bei Eintreten eines Störfalls.

\section{Aufgabenstellung}

Die aus einer Vielzahl angepasster Einzelmaßnahmen bestehenden Schutzkonzepte erfordern häufig geeignete Sorptionsmittel zur Abbindung und Entfernung von Ölen aus aquatischen Systemen, die einfach in ihrer Anwendung und gut lagerfähig sind.

Im Rahmen der Kooperation der Technischen Hochschule Wildau und der Universität für Chemische Technologie und Metallurgie Sofia wurden bislang Komposite auf der Basis von Holz und verschiedenen Bindemitteln auf der Basis von Polyestern oder Polyurethanen im Rahmen von Diplomarbeiten entwickelt [Evtimova et al. 2004]. Komposite aus Holz und Polyurethan-Prepolymeren auf der Basis von Recyclatpolyolen sind ebenfalls untersucht worden [Behrendt et al. 2004]. Dieses Verfahren sind auch auf pulverförmige bzw. granulierte Polyurethan-Hartschaum-Reststoffe angewendet worden, wofür feste Polyurethan-Bindemittel mit geeignetem Schmelzpunkt und Klebverhalten entwickelt wurden [Georgieva et al. 2005].

Auf Grund der Hydrophobierung der Holzoberflächen bei gleichzeitigem Erhalt der Ölbindekapazität erscheinen solche Systeme gut geeignet als Sorptionsmittel und sollten daher einer eingehenden Untersuchung unterzogen werden. Hierbei soll insbesondere die Wasseraufnahmefähigkeit als Kriterium der Instabilität derartiger Komposite und die Ölaufnahmefähigkeit als Maß für die Sorptionskapazität erfasst werden. Mit einem einfachen Ansatz zur Verteilung der aktiven Zentren der Komponenten soll die Hydrophobierung geometrisch begründet werden.

\section{Ergebnisse}

Die Sorptionsmittel wurden als Komposite von zellulosehaltigem Material mit einem PUR-Prepolymer als Bindemittel formuliert.

Die Herstellung der Prepolymere erfolgte nach der in [Georgieva et al. 2005] beschriebenen Methode, wobei als Polyol ein Aromatisches Polyesterpolyol (APP) mit einer $\mathrm{OH}-\mathrm{Zahl}$ von $320 \mathrm{mg} \mathrm{KOH} / \mathrm{g}$ und einer Viskosität von $3180 \mathrm{mPas}\left(25^{\circ} \mathrm{C}\right)$ verwendet wurde. Ein solches APP wurde nach [Behrendt et al. 1998] durch die Umsetzung von Polyethyleterephtalat (PET) mit Diethylenglycol (DEG) erhalten.

Das APP wurde mit einem Poly-Isocyanat (p-MDI) umgesetzt; als günstig erwies sich ein NCO : $\mathrm{OH}$ Ver- 
$50 \mu \mathrm{m}$

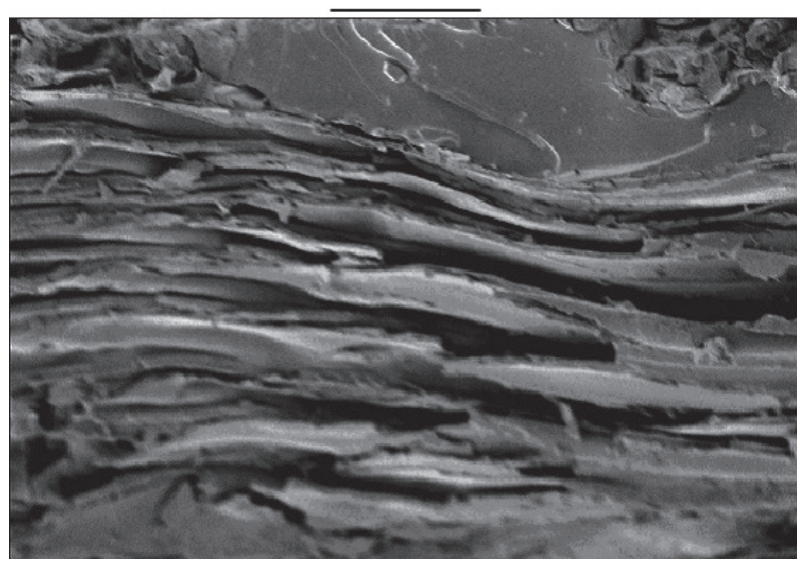

Abb. 2: Elektronenmikroskopische Aufnahme eines Holz-Prepolymer Komposits an der Bruchkante eines Kryobruchs

füllt und mit definierten Mengen Öl versetzt. Anhand des Durchbruchs der Ölfront mit dem Abtropfen des ersten Öltropfens am Boden wird die Kapazität ermittelt, die Ergebnisse sind in Tabelle 2 dargestellt.

\begin{tabular}{|c|c|c|c|}
\hline \multirow[t]{2}{*}{ Komponenten } & \multirow{2}{*}{$\begin{array}{l}\text { Anteil } \\
{[\mathrm{Ma}-\%]}\end{array}$} & \multicolumn{2}{|c|}{ Sorptionskapazität [göı/gSorptionsmittel] } \\
\hline & & Pulver & Presskörper \\
\hline Holzspäne & 95 & \multirow{2}{*}{1,26} & \multirow{2}{*}{1,08} \\
\hline Prepolymer & 5 & & \\
\hline Abrieb & 90 & \multirow[b]{2}{*}{2,61} & \\
\hline Holzspäne & 10 & & \\
\hline Holzspäne & 85 & \multirow{3}{*}{1,64} & \multirow{3}{*}{1,35} \\
\hline Abrieb & 5 & & \\
\hline Prepolymer & 10 & & \\
\hline Abrieb & 90 & \multirow[b]{2}{*}{1,50} & \multirow[b]{2}{*}{1,50} \\
\hline Prepolymer & 10 & & \\
\hline
\end{tabular}

Tabelle 2: Absorptionskapazität der Kompositmaterialien für Mineralöl

Die ermittelten Eigenschaften liegen letztlich in der molekularen räumlichen Struktur der wesentlichen Komponenten begründet. Zur Veranschaulichung wurden hierzu mittels einer Simulation Modellvorstellungen zur Verteilung der reaktiven Zentren erarbeitet, die in Abbildung 3 dargestellt sind.

nahme in Abb. 2 zeigt die Verdichtung in Pressrichtung und eine resultierende schichtartige Struktur des Sorptionsmittels.

Zur Charakterisierung der Eignung als Sorptionsmittel wurde die Ölbindekapazität in Kombination des o. g. Holzmaterials mit einem pulverförmigen Faserabrieb (ca. 50 Ma-\% Wolle, ca. 25 Ma-\% Viskose und ca. 25 Ma-\% Polyamid), einem Reststoff der Textilindustrie, ermittelt. Als Öl wurde ein Mineralöl der Dichte $0,85 \mathrm{~kg} / \mathrm{l}$ verwendet.

Mit der in [Nenkova et al. 2008] beschriebenen dynamischen Methode wird ein Glasrohr mit Material be-

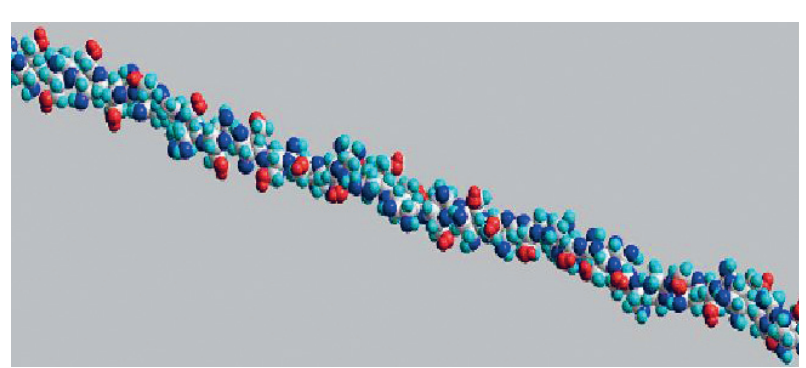

Abb. 3a: Ausschnitt aus einem Zellulose-Molekül (ca. 20 Monomere); weiß $=$ Kohlenstoff, blau $=$ Sauerstoff, cyan $=$ Wasserstoff, grün $=$ Stickstoff, rot = reaktive funktionelle Gruppen $(-\mathrm{OH}$ bzw. $-\mathrm{NCO})$. 


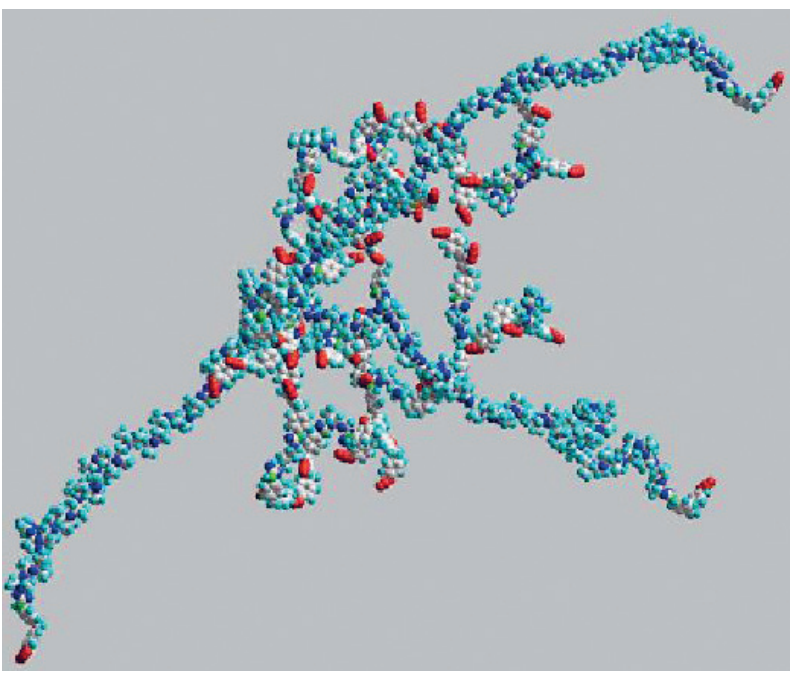

Abb. 3b: Vereinfachte Darstellung eines Prepolymer-Gemisches; weiß = Kohlenstoff, blau = Sauerstoff, cyan = Wasserstoff, grün = Stickstoff, rot = reaktive funktionelle Gruppen $(-\mathrm{OH}$ bzw. $-\mathrm{NCO})$.

Die Abbildung 3b zeigt ein Prepolymer, wie es aus recycliertem PUR-Kaltweichschaum [Georgieva et al. 2005] durch Umsetzung mit MDI erhalten wird. Es wurden dazu die langkettigen Polyetherpolyole (Molmasse ca. 5000) und Dipropylenglycol mit dem Isocyanatgemisch Polymer-MDI im ca. 3-fachen Überschuss eingesetzt. Die im Prepolymer ebenfalls vorhandenen Oligoharnstoffe und Amine sind aus Gründen der Übersichtlichkeit weggelassen worden.

\section{Diskussion}

Die Untersuchungen zur Quellung in Wasser zeigen ein anisotropes Quellverhalten mit nahezu ausschließlicher Quellung in Richtung der bei der Herstellung durch den Pressvorgang reduzierten Probendicke, wobei mit steigendem Bindemittelanteil die Proben zunehmend hydrophobiert werden. Bereits mit $15 \%$ Prepolymer weisen die Materialien eine Stabilität über einen Tag auf, mit 20 \% Prepolymer können die Materialien über 4 Wochen im Wasser verbleiben ohne zu zerfallen.

Das ermittelte Ölrückhaltevermögen war stets höher als das Eigengewicht des Absorptionsmittels. Der Einsatz eines Bindersystems und der Pressvorgang reduzieren allerdings merklich die Sorptionskapazität. Die Beimengung geringer Mengen von Faserabrieb wirkt einer Reduktion der Sorptionskapazität entgegen.

Das als Bindemittel für zellulosehaltige Materialien eingesetzte Prepolymer weist Abstände zwischen den reaktiven NCO-Gruppen im Bereich von ca. $3 \mathrm{~nm}$ auf und ist damit im Vergleich mit der Dichte an primären $\mathrm{OH}-$ Gruppen von Zellulose in der Lage, nur einzelne, relativ weit voneinander entfernte chemische Bindungen auszubilden. Dies ist offensichtlich hinreichend zur Herstellung von Kompositmaterialien, die in wässrigen Medien für einige Tage stabil sind und eine beträchtliche Kapazität zur Aufnahme von Mineralölen aufweisen.

\section{Ausblick}

Für zellulosehaltige Materialien, die über eine Stützstruktur verfügen, bieten sich neben der Anwendung als Absorptionsmittel weitere interessante Anwendungspotenziale, z. B. als Filtermaterial in biologischen Abluftreinigungsanlagen. Reiner Rindenmulch oder andere Schüttgüter scheiden hier häufig wegen des schwer zu kontrollierenden Druckverlusts, des Eigengeruchs und einer geringen Standzeit aus. Eine gezielte Modifizierung sowohl der Oberflächeneigenschaften für eine biologische Besiedlung als auch der Körnung für die verfahrenstechnischen Parameter ist mit der vorgestellten Technologie prinzipiell zugänglich.

\section{Acknowledgement}

Dank gilt der NATO für die großzügige Unterstützung der Entwicklung der Zusammenarbeit der bulgarischen und deutschen wissenschaftlichen Einrichtungen mit einem Collaborative Linkage Grant unter dem Förderkennzeichen CLG 982877. 


\section{Literaturverzeichnis}

Amini, A.; Bollaert, J.; Boillat, J.-L.; Schleiss, A. J. (2008): Dynamics of low-viscosity oils retained by rigid and flexible barriers Ocean Engineering, 35, 1479-1491.

Behrendt, G.; Lobeda, A.; Pohl, M. (1998): Verfahren zur Herstellung von Polyesteralkoholen sowie Polyesteralkohole, WO 99/54380 DE-OS 19918650.

Behrendt, G.; Hunger, H.-D.; Madjanova, R.; Georgieva D.; Evtimova R. (2004): Polymer-Holz Komposite mit Recyclaten, Vortrag auf dem 4. Beckmann-Kolloquium, Wismar 04.06.2004.

Elshorbagy, W.; Elhakeem, A.-B. (2008): Risk assessment maps of oil spill for major desalination plants in the United Arab Emirates Desalination, 228, 200-216.

EU (1978), Entschließung des Rates vom 26. Juni 1978 zur Erstellung eines Aktionsprogramms der Europäischen Gemeinschaften auf dem Gebiet der Überwachung und Verringerung der Ölverschmutzung des Meeres Amtsblatt Nr. C 162 vom 08.07.1978, $0001-0004$.

Evtimova, R.; Madjanova, R.; Georgieva, D.; Hunger H.-D.; Behrendt, G. (2004): Polymer Wood Composites Based on Recycling Products from Condensation Polymers, Paper presented at the Conference »Manufacturing and Management in the 21st Century«, Ohrid, September 2004.

Exxon Valdez Oil Spill Trustee Council (2008), Statusreport.

Georgieva, D.; Nenkova, S.; Hunger, H.-D.; Behrendt, G. (2005): Kompositwerkstoffe aus Kombination von Polyurethan-Reststoffen, Wissenschaftliche Beiträge 2005 der Technischen Fachhochschule Wildau 2005, 79-84.

Homan, A. C.; Steiner, T. (2008): OPA 90's impact at reducing oil spills, Marine Policy 32, 711-718.

Huijer, K. (2005): Trends in Oil Spills from Tanker Ships, 28th Arctic and Marine Oilspill Program (AMOP) Technical Seminar, 7-9 June 2005, Calgary, Canada.

König, G. (2007) in: GASWINNERs 2/2006

Li, Y. (2007): Control of spatial discretisation in coastal oil spill modelling International Journal of Applied Earth Observation and Geoinformation, 9, 392-402 (2007).

Nenkova, S.; Georgieva, D.; Garvanska, R.; Gancheva, V.; Herzog, M. (2008): Development and study of wood polymer sorbents for cleaning of oil polluted water, Journal of the University of Chemical Technology and Metallurgy Sofia, 43, 2, 2008, 217-222.

Ornitz, B. E.; Champ, M. A. (2002): Oil spills first principles: prevention \& best response, Amsterdam, Elsevier Science Ltd.

Schramm, W. (1972): Untersuchungen über den Einfluß von Ölverschmutzungen auf Meeresalgen, Marine Biology 14, 189-198.

United Nations Environment Programme (1993) Report No. 50.

Vanem, E.; Endresen, O.; Skjong, R. (2008): Cost-effectiveness criteria for marine oil spill preventive measures Reliability Engineering and System Safety, 93, 1354-1368.

\section{Autoren}

Dr. rer. nat. Michael Herzog

TH Wildau [FH]

Fachbereich Ingenieurwesen/Wirtschaftsingenieurwesen

Tel. + 493375 508-354

michael.herzog@tfh-wildau.de

\section{Prof. Dr. habil. Sanchi Nenkova}

University of Chemical Technology and Metallurgy

$8 \mathrm{Kl}$. Ohridski bul.

1756 Sofia, Bulgaria

Tel. + 35926254101

nenkova@uctm.edu

\section{Prof. Dr. habil. Radka Garvanska}

University of Chemical Technology and Metallurgy

8 Kl. Ohridski bul.

1756 Sofia, Bulgaria

Tel. + 359897461949

garvanska@uctm.edu

\section{Dr. rer. nat. Valeria Gancheva}

Institute of Polymers

Bulgarian Academy of Science

Acad. G. Bontchev str. 103 A

1113 Sofia, Bulgaria

Tel. + 35928700309

valeriag@polymer.bas.bg 